\title{
Cardiovascular disease in the literature: A selection of recent original research papers
}

\author{
Wael A. AlJaroudi, MD, FASNC, ${ }^{\text {a }}$ and Fadi G. Hage, MD, FAHA, FACC, MASNC ${ }^{b, c}$ \\ a Division of Cardiovascular Medicine, Clemenceau Medical Center, Beirut, Lebanon \\ b Division of Cardiovascular Disease, Department of Medicine, University of Alabama at \\ Birmingham, Birmingham, AL \\ c Section of Cardiology, Birmingham Veterans Affairs Medical Center, Birmingham, AL
}

Received Apr 14, 2020; accepted Apr 15, 2020

doi: $10.1007 / \mathrm{s} 12350-020-02153-\mathrm{w}$

Potential Effects of Coronaviruses on the Cardiovascular System: A Review. JAMA Cardiol. https://doi. org/10.1001/jamacardio.2020.1286

Coronavirus disease 2019 (COVID-19), caused by severe acute respiratory syndrome coronavirus 2 (SARS-CoV-2), has been declared a pandemic with devastating effects on the entire world. Madjid et al. from the University of Texas Health Science, Houston, Texas review current information on the virus and COVID-19 and its effects on the cardiovascular system. Coronaviruses, classified in 4 groups, were first identified in humans in the 1960s with 4 types (all from the $\alpha$ and $\beta$ classes) are endemic in humans usually causing mild and self-limiting upper respiratory infections accounting for $15 \%-30 \%$ of common colds. Both SARS (caused by SARS-CoV) and Middle East respiratory syndrome (MERS, caused by MERS-CoV) caused outbreaks (in 2002 and 2012, respectively) that had higher fatality rates but were much less widespread than COVID-19. SARS-CoV-2 is genetically more similar to SARS-CoV (82\%) than MERS-CoV (50\%). The virus has a mean incubation period of 5.2 days $(95 \% \mathrm{CI}, 4.1$ 7.0 days), with the 95th percentile of the distribution at 12.5 days according to one study and up to 14 days according to another. The World Health Organization reported a global mortality rate of $3.4 \%$ but importantly, this rate varies by location, intensity of transmission, variations of care, presence of comorbidities (including cardiovascular disease), advanced age, and likely other

Reprint requests: Fadi G. Hage, MD, FAHA, FACC, MASNC, Division of Cardiovascular Disease, Department of Medicine, University of Alabama at Birmingham, Lyons Harrison Research Building 306, 1900 University BLVD, Birmingham, AL 35294; fadihage@uab.edu

J Nucl Cardiol 2020;27:712-24.

$1071-3581 / \$ 34.00$

Copyright (c) 2020 American Society of Nuclear Cardiology. factors. The primary symptoms of COVID-19 are fever, cough, and shortness of breath. Acute cardiac injury determined by elevated high-sensitivity troponin levels is commonly observed in severe cases and is strongly associated with mortality, complications such as acute respiratory distress syndrome, arrhythmia, renal injury, and coagulopathy. There have been reported cases of viral infiltration of the myocardium causing myocarditis. Myocardial injury can also be caused by ischemia. It is important to note that COVID-19 in addition to inducing new cardiac pathologies (such as myocarditis) may also exacerbate pre-existing cardiovascular disease. Finally, some of the investigational therapies for COVID-19 may have cardiovascular effects. Current treatment, until investigational therapies have been shown to be effective, is supportive care and treatment of complications. The article offers a wonderful review of current knowledge on COVID-19 as well as a review of SARS and MERS which provide further insights on the effects of coronaviruses on the cardiovascular system.

Association of Public Health Interventions With the Epidemiology of the COVID-19 Outbreak in Wuhan, China. JAMA 2020. https://doi.org/10.1001/jama.2020. 6130.

Background: Coronavirus disease 2019 (COVID19) has become a pandemic. Pan An et al. from Tongji Medical College, Wuhan, China, sought to assess whether different public health interventions during 5 consecutive periods could improve control of the outbreak in Wuhan: Period 1 (Dec 8-Jan 9)-No intervention; Period 2 (Jan 10-22)-Massive human movement due to Chinese New Year; Period 3 (Jan 11Feb 1)-implementation of home quarantine, traffic restriction, cordon sanitaire; Period 4 (Feb 2-16)-Centralized quarantine; Period 5 (Feb 17-march 8)- 
Universal symptoms survey. Individual-level data on laboratory-confirmed COVID-19 cases were extracted from the municipal Notifiable Disease Report System. The rates of laboratory-confirmed COVID-19 infections and the effective reproduction number of SARS-CoV-2 (an indicator of secondary transmission) were calculated and compared for each of the 5 periods.

Findings: Among 32583 laboratory-confirmed COVID-19 cases (median age 57 years, 52\% women), the daily confirmed case rate peaked in the third period and declined afterward across geographic regions, gender, and age groups. Local health care workers were significantly more affected than the general population (130.5 per million people vs 41.5 per million people) in the early periods, an indicator of nosocomial infection due to lower awareness and inadequate use of personal protective equipment in the early periods, and quickly decreased in the subsequent periods. Severe and critical cases decreased from $53.1 \%$ to $10.3 \%$ over the 5 periods.

Significance: The institution of interventions including cordons sanitaire, traffic restriction, social distancing, home quarantine, centralized quarantine, and universal symptom survey was temporally associated with reduction of new confirmed cases and secondary transmission of COVID-19 across age groups, sex, and geographic regions. These findings may be valuable in the current efforts to combat the global pandemic of COVID-19 as they underscore the importance of public awareness and early implementation of multifaceted public health interventions. The limited testing in the first periods as well as under-reporting of asymptomatic cases probably underestimated the numbers. Also, because of the implementation of multiple public interventions at the same time, the impact of each individual strategy cannot be inferred.

Cardiac troponin I in patients with coronavirus disease 2019 (COVID-19): Evidence from a metaanalysis. Prog. Cardiovasc. Dis. https://doi.org/10.101 6/j.pcad.2020.03.001.

Background: Patients with cardiovascular disease are at higher risk for complications and death with COVID-19. Lippi et al. from University of Verona, Italy performed a meta-analysis of studies that investigated the association between cardiac troponin and severity of COVID-19.

Results: After excluding non-pertinent paper, 4 studies were included. All 4 studies used cardiac troponin I, and all except one used high-sensitivity assays. All 4 studies were from China and included a total of 341 patients (36\% with severe disease). The clinical outcome was defined as intensive care unit admission in
2 studies, acute respiratory distress syndrome in one study, and death in one study. Cardiac troponin I was significantly higher in patients with severe COVID-19 compared to those without severe disease (standardized mean difference $25.6 \mathrm{ng} / \mathrm{L} ; 95 \% \mathrm{CI}, 6.8-44.5 \mathrm{ng} / \mathrm{L})$. There was significant heterogeneity between studies $\left(I^{2}\right.$, 98\%; $P<.001)$.

Significance: Elevated cardiac troponin in patients with COVID-19 indicates the presence of cardiac injury which is associated with more severe disease, higher rate of complications, and higher likelihood of death. Whether stratifying treatment strategies by troponin elevation will be useful in management of these patients remains to be seen.

Epidemiology of Covid-19 in a Long-Term Care Facility in King County, Washington. $N$ Eng J Med 2020. https://doi.org/10.1056/nejmoa2005412.

Background: The vulnerability of long-term care facilities residents to respiratory disease outbreaks is well known. Due to their advanced age and underlying chronic health conditions, they are at increased risk for severe complications and poor outcomes from outbreaks of COVID-19. After the identification on February 28, 2020 of a confirmed case of COVID-19 in a skilled nursing facility in King County, Washington, McMicheal $\mathrm{T}$ et al. from the Public Health-Seattle and King County aided by the Centers for Disease Control and Prevention, launched an investigation with contact tracing, quarantined exposed persons, isolated confirmed and suspected cases in an effort to contain the disease.

Findings: As of March 18 2020, less than 3 weeks from the first-identified case, a total of 167 confirmed cases of COVID-19 (median age 72 years, $67 \%$ female) were found to be epidemiologically linked to the facility; they included 101 residents, 50 health care personnel, and 16 visitors. The majority of cases among residents had respiratory symptoms, and only $7 \%$ were asymptomatic. The hospitalization rates for facility residents, visitors, and staff were $55 \%, 50 \%$, and $6 \%$, with corresponding case fatality rates of $34 \%, 0 \%$, and $6 \%$. In addition, a total of 30 long-term care facilities with at least one confirmed case of Covid-19 had been identified in King County were identified within this short period.

Significance: The study underscores the high transmission rate of COVID-19, and the vulnerability of long-term care facility residents with one-third case fatality rate. Staff working in multiple facilities while ill and the transfer of patients from one facility to another are potential causes of spread. Proactive steps to identify and exclude potentially infected staff and visitors, and implementation of appropriate infection prevention and 
control measures, are necessary to halt the spread of the disease. On March 10, the governor of Washington implemented mandatory screening of health care workers and visitor restrictions for all licensed nursing homes and assisted living facilities.

Compassionate Use of Remdesivir for Patients with Severe Covid-19. N Engl J Med 2020. https://doi. org/10.1056/nejmoa2007016.

Background: Coronavirus disease (COVID-19) has become a pandemic with alarming transmission and death rates. Grein J et al. from Cedars-Sinai Medical Center, Los Angeles, investigated the compassionate use of Remdesivir, a nucleotide analogue prodrug that inhibits viral RNA polymerases with in vitro activity against SARS-CoV-2, in 61 patients hospitalized with COVID-19. Inclusion criteria included patients with confirmed SARS-CoV-2 infection, oxygen saturation less or equal to $94 \%$ on room air or the requirement of oxygen support. Patients received a 10-day course of Remdesivir, consisting of $200 \mathrm{mg}$ administered intravenously on day 1 , followed by $100 \mathrm{mg}$ daily for the remaining 9 days of treatment.

Findings: Between January 25 and March 7 2020, 53 patients ( 22 in the USA, 22 in Europe or Canada, and 9 in Japan) received compassionate use of Remdesivir and had available post-treatment data for analysis.
Almost $65 \%$ of the patients were on high oxygen support measures at baseline ( $57 \%$ ventilators, $8 \%$ extracorporeal membrane oxygenation). After a median follow-up of 18 days, two-third had an improvement in oxygensupport class, with more than half of those on ventilators getting extubated. A total of 25 patients (47\%) were discharged. The overall mortality rate was $13 \%(7 / 53)$ and was significantly higher for those receiving invasive ventilation $(6 / 34,18 \%)$ compared to those not on ventilator $(1 / 19,5 \%)$.

Significance: In this cohort of sick patients hospitalized for severe COVID-19 and requiring oxygen support, the compassionate use of Remdesivir resulted in clinical improvement of two-third of patients. The exclusion of $8 / 61$ patients due to unavailable data posttreatment, and the lack of a control group are significant limitations of this study. Randomized, placebo-controlled trials are needed to validate the results of this small size uncontrolled study, and those trials are ongoing. The challenge of conducting fast-randomized clinical trials trying to fight a pandemic is a global challenge and requires putting everyone's resources and expertise.

Publisher's Note Springer Nature remains neutral with regard to jurisdictional claims in published maps and institutional affiliations. 